\title{
HABILIDADES EMOCIONALES Y PROFESIONALIZACIÓN DOCENTE PARA LA EDUCACIÓN INCLUSIVA EN LA SOCIEDAD EN RED
}

\author{
Alicia Peñalva Velez \\ Jose Javier López-Goñi \\ Jesús Barrientos González \\ Universidad Pública de Navarra
}

\begin{abstract}
RESUMEN: Los retos que la sociedad red y el desarrollo del modelo educativo inclusivo plantean al profesorado no hacen sino incrementar los factores estresores de la profesión docente. Frente a ello, el engagement se perfila como el estado cognitivo-afectivo que, vinculado al desarrollo de las habilidades emocionales, sirve al profesorado como estrategia de afrontamiento del estrés laboral. Cada vez más estudios indican la necesidad de desarrollar programas de formación en habilidades emocionales e inteligencia emocional, para la mejora de la salud y bienestar del profesorado. A través de una revisión bibliográfica de la literatura especializada se busca establecer la relación que existe entre los conceptos de burnout, engagement, inteligencia emocional y habilidades emocionales. Se constata que cada vez son más los trabajos que avalan la relación existente entre ellos, y que apuestan por la formación de los docentes en el ámbito específico de la inteligencia emocional como herramienta adecuada para la gestión positiva del burnout hacia el engagement. En consecuencia, es necesario establecer programas de formación para el profesorado, que le dote de las herramientas emocionales necesarias.
\end{abstract}

PALABRAS CLAVE: Burnout, engagement, inteligencia emocional, competencias emocionales, profesión docente.

\section{EMOTIONAL SKILLS AND TEACHERS TRAINING FOR INCLUSIVE EDUCATION IN NETWORK SOCIETY}

\footnotetext{
ABSTRACT: Network society and inclusive education are now two major challenges for teachers. Both increases the stress factors of the teaching profession. The engagement is proposed as an affective-cognitive status which linked to the development of emotional skills. It can be a tool for teachers to cope with job stress or burnout. A lot of studies indicate the need to develop
} 
emotional skills and emotional intelligence training programs to improve teachers welfare. A literature review was performed to establish the connection between the concepts of burnout, engagement, emotional intelligence and emotional skills. It is noted that many studies show the relationship between the concepts. They indicate the need to train teachers in emotional intelligence to be able to walk from the burnout to engagement. It means that is necessary to design emotional intelligence training programs for teachers to give them the necessary emotional tools to develop their profession.

KEYWORDS: Burnout, engagement, emotional intelligence, emotional skills, teaching profession.

Recibido: 31/03/2016

Aceptado: 18/07/2016

Correspondencia: Alicia Peñalva Vélez, Universidad Pública de Navarra, Facultad de Ciencias Humanas y Sociales, Departamento de Psicología y Pedagogía, Campus de Arrosadía, 31006 Pamplona, Navarra (España). Email: alicia.penalva@unavarra.es.

\section{INTRODUCCIÓN}

La profesión docente se ve abocada a transformaciones cada vez mayores, que plantean la necesidad de revisar las competencias que el profesorado debe poseer (Asensio, Álvarez, Vega y Rodríguez, 2012; Bozu y Canto, 2009; Pérez-Gómez, 2010; Marina, Pellicer y Manso, 2015; Tello y Aguaded, 2009). En este sentido Marina, Pellicer y Manso (2015) establecen con claridad aquellas competencias que resultan ahora más necesarias para los docentes (p. 14-17):

(a) Una idea clara del funcionamiento de la inteligencia humana, de sus capacidades y posibilidades (...). (b) Organizar y animar situaciones de aprendizaje (...). (c) Gestionar el progreso en el aprendizaje (...). (d) Saber enfrentarse a conflictos y problemas de convivencia y ayudar a que los alumnos sepan revolverlos (...). (e) Saber trabajar en equipo con el resto del claustro (....). (f) Dar importancia a la relación con las familias (...). (g) Utilizar las nuevas tecnologías dentro del aula (...). (h) Adaptación a entornos multiculturales y necesidad del bilingüismo (...). (i) Enfrentarse competentemente a los dilemas éticos de la profesión (...). (j) Estar dispuesto a aprender siempre (...).

Todas ellas se pueden agrupar desde nuestro punto de vista en dos grandes bloques de análisis, identificados también por los autores, y que giran en torno a dos necesidades: (1) que la escuela se abra a la sociedad red para aprovechar sus recursos, teniendo en cuenta (entre otras) la potente presencia de las nuevas tecnologías, y (2) la aspiración a una escuela inclusiva dirigida a impulsar el talento de cada alumno (Marina, Pellicer y Manso, 2015). En otras palabras, que la escuela afronte los cambios que a nivel escolar se derivan de la sociedad red (Castells, 2006), y que aborde las estrategias necesarias para el desarrollo de escuelas inclusivas (Echeita, 2016). 
La revolución tecnológica producida a escala mundial desemboca en el nacimiento de la actual sociedad de la información y de la tecnología. Una Sociedad que se puede definir por todas las actividades de recepción, generación, gestión y uso de datos que en ella se producen a través de las Tecnologías de la Información y la Comunicación (TIC). Todas ellas requieren de una formación o alfabetización específica (Área, 2012; Área et al., 2015; De Pablos, 2010; Fernández-Montalvo, Peñalva e Irazabal, 2015) tanto de los nativos como de los inmigrantes digitales (Prensky, 2010). En este contexto se habla del concepto de ciudadanía digital o ciberciudadanía, entendido como una nueva forma de ser en el mundo globalizado y conectado mediante las TIC. El concepto hace referencia concretamente al uso eficaz de las redes de comunicación como vía habitual de comunicación y a la capacidad de usar Internet como vía de participación democrática (Pineda, 2011). Efectivamente, en la actual sociedad de la información y la tecnología, las TIC son la vía más utilizada para el procesamiento y transmisión de gran parte de la información que se maneja en todos los ámbitos y edades (Castells, 2008, 2005 y 1997).

En los últimos años la Web 2.0 ha cambiado las reglas de juego establecidas en el ámbito de las relaciones comunicativas basadas en los medios de comunicación convencionales (Aparici, 2010; Área, Gutiérrez y Vidal, 2012; Área y Pessoa, 2012). Las nuevas aplicaciones y técnicas que ofrece son el punto de partida para un nuevo concepto de aprendizaje y de comunicación, un nuevo concepto de socialización. Es lo que Castells (2005) denomina sociedad de la información, enmarcada, como afirma Lévy (2007) en su respectiva cibercultura. Hemos pasado de una cultura "sólida" propia del siglo XIX a una cultura "líquida" característica del siglo XX, en la que el conocimiento es completamente inestable y fluye a cada momento (Bauman, 2006). Es una cultura multimodal (Área, Gros y Marzal, 2008; Área et al., 2012), que demanda nuevos procesos de alfabetización de la ciudadanía, más allá de la mera formación en herramientas tecnológicas.

Nos encontramos ante lo que Van Dijk (2006) y Castells (2006) definen como la sociedad red, o "network society". Ésta se caracteriza por ser un tipo de sociedad que organiza sus relaciones en redes digitales, que van sustituyendo a las tradicionales redes de comunicación cara a cara (Crespi y Cañabate, 2010). Las estructuras sociales y las actividades claves son organizadas alrededor de redes digitales, que se organizan y existen de forma exclusivamente digital (Castells, 2006). Las repercusiones que esto tiene a nivel educativo son grandes, puesto que, como afirma Uztarroz (2012, p. 139-140): "tanto el currículo como la propia metodología escolar quedan totalmente obsoletos para procurar a los alumnos la adquisición de las habilidades requeridas en los nuevos entornos sociales y profesionales (...)". Un problema que sin embargo no es nuevo, como lleva tiempo señalando el paradigma de la educación inclusiva (Armstrom, 2000; Beltrán, 2012). La educación, desde el punto de vista de la inclusión, se define como un proceso permanente dirigido a ofrecer una educación de calidad para todos (UNESCO, 2008). Y la calidad significa, desde la perspectiva de la inclusión, llegar a todo el alumnado de forma eficiente, promoviendo el desarrollo cognitivo del educando y estimulando los valores y actitudes de la ciudadanía responsable (Beltrán, 2012; UNESCO, 2008).

Esto implica, como señala Echeita (2016), un proceso de reestructuración escolar que comprende la puesta en marcha de diferentes procesos de innovación y mejora, 
fundamentados en dos criterios difíciles de armonizar (Beltrán, 2012): la calidad educativa y la diversidad de los alumnos. La educación inclusiva como proceso implica cambios metodológicos profundos tanto en el centro como en el aula. Son cambios que se centran en los procesos de enseñanza y evaluación y que se concretan en: (1) la adaptación de objetivos y contenidos a los diferentes niveles educativos, (2) un profesorado competente, (3) el desarrollo de una metodología activa y participativa, (4) la puesta en marcha de apoyos internos y externos efectivos, (5) la puesta en marcha de procedimientos adecuados de evaluación, y (6) una estructura organizativa de centro estable (Ainscow, 2001; Arnaiz, 2003; Booth, Ainscow y Kingston, 2006; Echeita, 2006; Montolío y Cervellera, 2008). Que se produzcan estos cambios dependerá de factores que se agrupan en tres niveles: (a) factores personales del maestro -formación inicial y permanente, experiencia profesional, creencias personales, y actitudes-, (b) factores internos del aula -organización del espacio y de las dinámicas de trabajo-, (c) factores internos de la escuela -relacionados con la política educativa, el ideario del centro, la financiación, y los recursos disponibles- (Booth et al., 2006; Echeita, 2006 y 2016; Beltrán, 2012).

Los principales retos que se plantean al profesorado en la sociedad red son dos. En primer lugar, el reto que por sí misma plantea la sociedad red a los docentes desde la perspectiva de la alfabetización digital. En segundo lugar, el reto que plantea el paradigma de la educación inclusiva desde la perspectiva de la construcción de una nueva concepción sobre la educación. En ambos casos se trata de abordar una reconfiguración del rol docente que implica, como señalan Almiron y Porro (2014, p. 5): "un cambio en las estrategias de enseñanza, (...) los educadores además de dominar los contenidos curriculares, deben conocer los procesos implicados en el aprendizaje que requiere la aplicación de métodos flexibles adaptados y basados en las necesidades de los alumnos (...)".

Desde el marco de la inclusión el profesorado debe evolucionar en tres dimensiones básicas: la creación de culturas de centro inclusivas, la elaboración de políticas inclusivas, y el desarrollo de prácticas inclusivas (Booth et al., 2006). Desde el marco de la sociedad red el profesorado se enfrenta ante el proceso de aprendizaje que comprende la adquisición de la competencia mediática (González-Fernández, Gozálvez y Ramírez, 2015), y ante el proceso de aprendizaje que implica su alfabetización digital (Área, 2015; Área et al., 2015). En este entorno docente, con estas características y variables condicionantes, las situaciones de estrés que se plantean al profesorado son una realidad señalada por distintos autores (Cornejo y Quiñonez, 2007; De la Torre, 2007; Durán, Extremera y Rey, 2010; Extremera, Rey y Pena, 2010). Ante ello, la educación emocional estructurada y explícita, es una forma de prevención primaria inespecífica (Bisquerra, 2005), con un doble efecto: la adquisición de habilidades de manejo del estrés, y la adquisición de habilidades docentes (Peñalva et al., 2013). Efectivamente, tal y como especifican los autores:

Hay dos motivos básicos por los que el profesorado necesita desarrollar las habilidades relacionadas con las competencias emocionales: el primero que tiene que ver con su bienestar emocional (Brotheridge y Grandey, 2002); el segundo que tiene que ver con su calidad docente y su papel como transmisor de conocimiento y como garante del bienestar del alumnado (Durlak, Weissberg, Dymnicki, Taylor y Schellinger, 2011; Muñoz de Morales, 2005) (Peñalva et al., 2013, p. 693). 
Por todo ello la finalidad principal de este artículo es constatar que cada vez más estudios indican la necesidad de desarrollar programas de formación en habilidades emocionales e inteligencia emocional, que prevengan el estrés en el profesorado, mejoren su salud y bienestar, y fomenten la calidad de su labor docente. Esta necesidad se constata en distintos estudios relacionados con la influencia de la educación emocional y la inteligencia emocional en el alumnado de distintos niveles educativos (Fernández-Berrocal y Ruiz, 2008a; Ferraguto y Fierro, 2012; Jiménez-Morales y López-Zafra, 2009). Sin embargo, tal y como afirman Pena y Extremera (2012, p. 609) "el interés por analizar la incidencia del síndrome (de burnout) en este colectivo (el docente) ha sido escaso en nuestro país". Para ello se realiza una revisión de los artículos publicados en idioma castellano en cuatro bases de datos, que abordan de manera específica los conceptos de inteligencia emocional, burnout, engagement y habilidades emocionales en el profesorado. La finalidad última es poder determinar cuáles pueden ser las bases para el diseño de un programa de formación en competencias emocionales para el desarrollo de la inteligencia emocional en el profesorado.

Los objetivos que se plantean en este artículo son dos: (1) realizar una revisión bibliográfica que muestre las investigaciones publicadas en castellano sobre los conceptos de burnout y engagement en el ámbito específico de la profesión docente. (2) Realizar una revisión bibliográfica que muestre cuáles de esas investigaciones ponen en relación los conceptos de competencias emocionales e inteligencia emocional, con los conceptos de burnout y engagement. Para ello se procederá a analizar de forma específica qué relación tienen con la profesión docente los conceptos de burnout, engagement, inteligencia emocional y habilidades emocionales. Se pretende identificar la relación existente, según las investigaciones analizadas, entre la formación en inteligencia emocional y competencias emocionales, y la mejora de la salud laboral y el bienestar del profesorado. Se busca así mismo comprobar la necesidad de desarrollar programas de formación en competencias emocionales y en inteligencia emocional en el profesorado. Todo ello en un total de cuatro bases de datos.

\section{Método}

Se seleccionaron un total de cuatro bases de datos para realizar la búsqueda bibliográfica: (1) ISOC, ciencias sociales y humanidades, sección psicología, (2) SCOPUS, (3) ProQuest humanidades y (4) PsycINFO (Tabla 1). Los términos introducidos para la búsqueda fueron un total de cinco, todo ellos en castellano: (a) inteligencia emocional, (b) inteligencia emocional profesorado, (c) burnout profesorado, (d) engagement profesorado y (e) habilidades emocionales (Tabla 1). En una primera fase se realizó una clasificación de los artículos en función de los títulos y de los autores. La muestra original de los artículos obtenidos en esta primera fase queda igualmente indicada en la Tabla 1. En una segunda fase, se realizó un análisis del contenido de los artículos, para asegurar que lo que en ellos se trabajaba se correspondía con la temática específica abordada en este estudio. Tras esta segunda fase la muestra total quedó formada por un total de 38 artículos. 
Tabla 1. Relación de artículos según bases de datos consultadas y términos de búsqueda empleados

\begin{tabular}{ccccc}
\hline & \multicolumn{4}{c}{ Bases de datos consultadas } \\
\cline { 2 - 5 } Términos de búsqueda & ISOC & SCOPUS & $\begin{array}{c}\text { PRO QUEST } \\
\text { (humanidades) }\end{array}$ & PsycINFO \\
\hline Inteligencia Emocional & 432 & 82 & 2310 & 93 \\
Inteligencia Emocional profesorado & 22 & 2 & 258 & 2 \\
Burnout profesorado & 39 & 7 & 126 & 4 \\
Engagement profesorado & 4 & 4 & 185 & 1 \\
Habilidades emocionales & 9 & 5 & 928 & 3 \\
\hline
\end{tabular}

\section{Resultados}

Se constata que el estudio del estrés en el ámbito de la salud laboral ha cobrado una gran importancia en los últimos años. Se considera que el estrés es un riesgo psicosocial laboral con una gran influencia en la salud de las personas que lo sufren (Chamarro, Cladellas, Longas y Riera, 2012). Se ha estimado que un $12 \%$ de los trabajadores europeos podrían padecer el síndrome de burnout, prevalencia relacionada directamente con el estrés docente (Demarzo, García, Gómez, Montero y Pereira, 2013). La profesión docente padece niveles de estrés importantes, mayores que en otros ámbitos laborales (Extremera y Pena, 2012), dato avalado por las diferentes memorias estatales anuales del defensor del profesor publicadas por el Sindicato Independiente de Educación (ANPE) (ANPE, 2014; ANPE, 2013 y ANPE, 2012).

El estrés laboral crónico supone un estado mental negativo y continuo respecto a la actividad laboral que aparece en personas que no padecen otras alteraciones patológicas (Extremera, Pena y Rey, 2012). En muchas ocasiones el estrés laboral crónico deriva en el síndrome de burnout (SB) como respuesta a ese estrés producido en el entorno laboral. Su origen está tanto a nivel organizacional como a nivel individual (Montoya, Moreno, y Zuluaga, 2012). Esto sucede igualmente en el ámbito educativo, en el que las causas se identifican como múltiples, y dependientes también de aspectos a nivel organizacional y a nivel interpersonal (Extremera et al., 2012). El concepto de "estar quemado" que derivó en el SB fue descrito por primera vez por Freuenberger (1974), quien lo definía como una sensación de fracaso y de existencia agotada o gastada derivada de una sobrecarga del trabajador" (Montoya et al., 2012). La definición clásica del SB es la propuesta por Maslach (1993) que lo identifica como "un síndrome psicológico de agotamiento emocional, despersonalización y reducida realización personal que puede ocurrir en individuos normales que trabajan con personas de alguna manera" (en Llorens y Salanova, 2008, p. 20-21).

Sobre la base de esta definición se puede entender que el SB posee tres dimensiones básicas: (1) el agotamiento emocional, que distancia a la persona del trabajo como un modo de afrontamiento de la sobrecarga laboral (Llorens y Salanova, 2008). (2) El cinismo o despersonalización, componente actitudinal y comportamental cínico e indiferente que hace al trabajador establecer distancia entre él mismo y las personas receptoras de su trabajo, ignorándolas activamente y considerándolas obje- 
tos impersonales (Gil-Monte y Peiró, 1999 y 1997; Montoya et al., 2012). (3) El sentimiento de ineficacia, que presenta elementos cognitivo-aptitudinales que se traducen en sentimientos de baja realización personal, incompetencia y fracaso (Gil-Monte y Peiró, 1999; Montoya et al., 2012). Además de ello, Gil-Monte y Peiró (1999) y Montoya et al., (2012) señalan tres modelos psicosociales que explican el origen de este síndrome: (1) la teoría sociocognitiva del yo, (2) la teoría del intercambio social y (3) la teoría organizacional. En primer lugar la teoría sociocognitiva establece que los pensamientos influyen en cómo las personas perciben y hacen su trabajo. En segundo lugar, el modelo basado en el intercambio social propone como causa del SB la experiencia de falta de equilibrio entre la percepción de esfuerzo e implicación laboral del trabajador y la percepción que de ello tiene la institución, los clientes receptores del trabajo y los compañeros. En tercer y último lugar, la teoría organizacional propone que la estructura, la cultura y el clima organizacional son variables importantes ya que pueden propiciar o no desgaste laboral en los trabajadores.

Aunque la investigación de manera habitual remarca la importancia de los factores organizacionales en el desarrollo del SB, cada vez son más los trabajos que inciden y remarcan los factores intraindividuales y personales (Extremera, Pena y Rey, 2010). Queda claro que las variables personales ejercen un papel muy significativo en la presencia o no del síndrome (Extremera, Durán y Rey, 2010). Se identifican un total de cuatro variables personales: la autoestima, la autoeficacia percibida, el optimismo disposicional y las habilidades emocionales. En primer lugar, la autoestima, entendida como un proceso afectivo autoevaluativo en el que la persona experimenta una autovaloración personal positiva (Extremera, Durán y Rey, 2010); en segundo lugar, la autoeficacia percibida como la creencia en la propia capacidad de organización y de poner en práctica las acciones necesarias para la consecución de los objetivos propuestos; (3) en tercer lugar, el optimismo disposicional, como expectativas generadas hacia la ocurrencia de resultados positivos en el futuro; y en cuarto y último lugar, las habilidades emocionales, cuyo desarrollo se considera fundamental para la adquisición de un adecuado afrontamiento.

Respecto al afrontamiento Montoya et al. (2012) indican que se puede definir como el modo en que el sujeto resiste y supera demandas excesivas para adaptarse a una nueva situación y restablecer el equilibrio. Folkman y Lazarus (1980) establecen dos tipos de afrontamiento: el afrontamiento centrado en el problema cuya finalidad es resolver la situación que está generando estrés; el afrontamiento centrado en la emoción por el que se regulan las emociones que se asocian a la situación causante del estrés (en Aluja, Buscarri y Blanchi, 2002). A estos dos Quass (2006) añade un tercero: el afrontamiento centrado en la evitación, que se manifiesta en conductas evasivas y evitativas del problema (en Montoya et al., 2012).

En los últimos años toma fuerza un nuevo planteamiento que estudia no tanto las disfunciones del ser humano como sus fortalezas y su funcionamiento óptimo (García-Renedo, Llorens y Salanova, 2005). En esta corriente se estudia el compromiso con el trabajo como una variable de bienestar personal (Cornejo y Quiñonez, 2007). Esta variable, también Ilamada engagement es definida por Maslach y Leiter (1997) como un estado positivo que se caracteriza por la energía, la implicación y la eficacia. El estudio y desarrollo de este constructo en Europa está más centrado en su carácter motivacional positivo, que lo presenta como un estado cognitivo-afectivo persistente 
en el tiempo, no focalizado en una conducta u objeto específico, e integrado por las dimensiones de Vigor, Dedicación y Absorción (Salanova et al. 2000). Como señalan Durán, Extremera, Montalbán y Rey (2005), el vigor se refiere al alto nivel de energía de la persona unido a un fuerte deseo de esfuerzo y de persistencia en las tareas que realiza. La dedicación hace referencia a los altos niveles de entusiasmo, inspiración, orgullo y reto ante el propio trabajo. Y la absorción por último, se relaciona con los altos niveles de concentración y de felicidad durante el desempeño de la actividad laboral. Es decir, las dimensiones vigor y dedicación en el engagement se consideran opuestas a las de agotamiento y cinismo en el burnout. La energía o activación es la responsable de que la persona se aproxime más al agotamiento o más al vigor.

La llave que transforma el burnout en engagement viene dada por una nueva concepción de la inteligencia. Desde finales del siglo pasado el concepto de inteligencia se ha ido transformando hacia un constructo más psicologicista. Con la aparición en los años 80 de la teoría de las Inteligencias Múltiples de Gardner y la Teoría Triárquica de Stenberg se asientan las bases para el trabajo por la definición de un nuevo concepto: la inteligencia emocional (Cabello, Fernández-Berrocal y Ruíz-Aranda, 2010; Pena y Repetto, 2008). El estudio de la Inteligencia Emocional (I.E.) es relevante por sus implicaciones beneficiosas en la salud física y mental de las personas (Martínez et al., 2014; Martínez, Piqueras y Ramos, 2010). De hecho se considera que la I.E. juega un papel muy importante en el autocontrol emocional y la capacidad adaptativa de los individuos para afrontar situaciones estresantes (Martínez et al., 2014). Tal y como señalan Cabello, Fernández-Berrocal y Ruíz-Aranda, (2010), el modelo de mayor rigor científico en este ámbito de la I.E. es del generado por Mayer y Salovey (1997). Los autores definen la I.E. como la capacidad (1) para percibir, valorar y expresar las emociones con exactitud; (2) para acceder y generar sentimientos que faciliten el pensamiento; (3) para entender la emoción y el conocimiento emocional; (4) y para regular las emociones y promover el crecimiento emocional e intelectual.

A partir de esta definición, el modelo que se desarrolla se basa en la definición de las tres habilidades que se consideran necesarias para el desarrollo saludable de la propia I.E. (Cabello, Fernández-Berrocal, y Ruíz-Aranda, 2010): la percepción emocional que supone el ser capaz de identificar las propias emociones y de expresarlas adecuadamente; la integración emocional, la forma en que las emociones percibidas y expresadas influyen en la cognición; y la regulación emocional, o capacidad para que los propios pensamientos sean promotores del crecimiento emocional, intelectual y personal. La evaluación de la propia I.E. es lo que Salovey y Mayer (1990) denominan como inteligencia emocional percibida, que no es otra cosa que el metaconocimiento que las personas tienen sobre sus propias habilidades emocionales, sus propias creencias, o sobre sus propias capacidades de atención, claridad y reparación emocional intrapersonal.

El desarrollo de estas habilidades señaladas por Cabello et al. (2010), e identificadas en el modelo de Salovey y Mayer (1990), permitirá el desarrollo a nivel intraindividual de la competencia emocional. El concepto de competencia emocional hace referencia a los conocimientos, capacidades, habilidades y actitudes que se consideran necesarios para comprender, expresar y regular de forma apropiada los fenómenos emocionales (Bisquerra y Pérez-Escoda, 2007). Las competencias emocionales se consideran predictoras de un mejor afrontamiento ante los sucesos vi- 
tales cotidianos, y se relacionan además con mayores niveles de bienestar y ajuste psicológico en niños y adultos (Mayer, Salovey y Caruso, 2002; Pyhältö, Pietarinen y Salmela-Aro, 2011; Pena y Extremera, 2012; Peñalva, López-Goñi y Landa, 2013; Salovey, Bedell, Detweiler y Mayer, 2000). También tienen una relación directa con el constructo de I.E. (Agulló, 2011; Ballester-Vila y Sánchez-Santamaría, 2011; Bisquerra y Pérez-Escoda, 2007; Sánchez, Fernández-Berrocal, Montañés y Latorre, 2008), definido como la habilidad para percibir, asimilar, comprender y regular las propias emociones (Martínez, Piqueras e Inglés, 2014).

Este estudio se ha basado en una búsqueda bibliográfica en lengua castellana de los artículos registrados en cuatro bases de datos. En base a este criterio se aprecian cuáles son las principales tendencias que se producen en el contexto español. En posteriores estudios sería necesario contar también con las tendencias desarrolladas en otros ámbitos internacionales (Marina, Pellicer y Manso, 2015).

\section{DisCuSIÓN Y CONCLUSIONES}

En esta revisión se ha constatado el interés de los investigadores en la influencia de la I.E. y el desarrollo de las competencias emocionales en el profesorado. Sin embargo, la mayoría de los estudios se centran en profesorado de secundaria, y esta situación no ha variado desde el año 2012 (Pena y Extremera). Por este motivo las evidencias encontradas en este estudio subrayan la necesidad de establecer líneas de intervención que disminuyan la prevalencia del estrés laboral en el profesorado, sobre todo en los diferentes niveles de la educación obligatoria (Peñalva et al., 2013). Las líneas de intervención no han de basarse únicamente en afrontar el estrés laboral docente, resulta necesario también fomentar sentimientos de compromiso hacia la labor educativa en los profesionales de la enseñanza (Durán, Extremera, Montalban y Rey, 2005). El profesorado requiere de herramientas emocionales adecuadas para poder afrontar la realidad educativa cotidiana (Fernández-Berrocal y Ruíz-Aranda, 2008). Para ello es necesario incluir el desarrollo socio-emocional como parte de su formación inicial y permanente (Cabello, Fernández-Berrocal, y Ruíz-Aranda, 2010; Peñalva et al., 2013). Es necesario que el profesorado desarrolle la competencia emocional para su propio bienestar personal, para poder ejercer con efectividad y calidad su actividad docente, y para el adecuado desarrollo emocional y social de su alumnado (Extremera, Fernández-Berrocal, y Durán, 2003; Extremera, Rey y Pena, 2010; Extremera y Pena, 2012). Tal y como afirman Castejón, Martínez y Pertegaz (2011) la adquisición de estos recursos se asocia directamente a la salud mental de los docentes.

Los autores entienden que un programa de formación emocional dirigido al profesorado debería estar basado en los trabajos de Salovey y Mayer (1990) y su modelo teórico sobre la I.E., considerado el de mayor rigor científico en este ámbito. El modelo se basa en una serie de habilidades necesarias para el desarrollo saludable de la propia I.E. (Cabello, Fernández-Berrocal y Ruíz-Aranda, 2010). Las tres habilidades que se identifican son (1) la percepción emocional que supone ser capaz de identificar las propias emociones y de expresarlas adecuadamente, (2) la integración emocional, la forma en que las emociones percibidas y expresadas influyen en la cognición y (3) la regulación emocional o capacidad para que los propios pensamientos sean promotores del crecimiento emocional, intelectual y personal. El desarrollo de estas 
habilidades dotará a la persona de la competencia emocional necesaria para afrontar las situaciones que pueden dar lugar al estrés laboral. Las competencias emocionales que desarrollaría el profesorado a partir del programa de formación estarían basadas en estas tres habilidades, expresadas en términos de dimensiones (Bisquerra, 2005):

1. La conciencia emocional: conocer qué es la conciencia emocional para identificar las propias emociones y las de los demás y fomentar una actitud de apertura y de escucha a la realidad emocional propia y de los otros.

2. La regulación de las emociones: el ser capaz de gestionar la propia realidad emocional adquiriendo habilidades para la tolerancia a la frustración, el control de la ira y habilidades de afrontamiento. Para ello es necesario el trabajo en algunas técnicas concretas como el diálogo interno, el control de estrés, las autoafirmaciones positivas...

3. Las habilidades socio-emocionales: competencias que facilitan las relaciones interpersonales, la escucha y la capacidad de empatía.

4. El concepto de fluir (flow): experiencia óptima, aprender a fluir.

Los recursos y estrategias que proporciona la educación emocional son de tipo conductual, cognitivo, emocional, y de interacción social (Peñalva, López-Goñi, García-Manso, 2016; Salmurri, 2004). La educación emocional tiene una función preventiva y de mejora de los estados emocionales, que se realiza a través del aprendizaje, el entrenamiento y la puesta en práctica de recursos y estrategias para amortiguar y minimizar las emociones excesivas o excesivamente negativas, y promover y aumentar las positivas (Salmurri, 2012). En este sentido estudios como los de Diekstra (2008), Durlak, Weissberg, Dymnicki, Taylor y Schellinger (2011) o Zins (2004), muestran evidencias empíricas acerca de la eficacia y beneficios de los programas de educación emocional. En esta misma línea Salmurri y Skoknic, (2005) indican que en una comunidad escolar, una intervención basada en la adquisición y entrenamiento de habilidades sociales, de interacción y de autocontrol emocional, podría mejorar la salud psicológica de profesores y alumnos.

\section{REFERENCIAS BIBLIOGRÁFICAS}

Agulló, M. J. (2011). Evaluación de la educación emocional en el ciclo medio de educación primaria. Revista de Educación, 354, 765-783.

Ainscow. M. (2001). Desarrollo de escuelas inclusivas. Ideas, propuestas y experiencias para mejorar las instituciones escolares. Madrid: Narcea.

Almirón, M. E. y Porro, S. (2014). Los docentes en la Sociedad de la Información: reconfiguración de roles y nuevas problemáticas. Revista Iberoamericana de Informática Educativa, 19, 17-31.

Aluja, A., Biscarri, J. y Blanchi, A. (2002). Síndrome de quemarse en el trabajo (Burnout) y estrategias de afrontamiento: un modelo de relaciones estructurales. Revista de Psicología en el trabajo y las organizaciones, 18(1) 57-74.

ANPE (2012). Memoria Estatal del Defensor del Profesor. Curso 2011-2012. Recuperado de: http://www.eldefensordelprofesor.es/wp-content/uploads/2015/11/ MEMORIA-DEFENSOR-2011.pdf. 
ANPE (2013). Memoria Estatal del Defensor del Profesor. Curso 2012-2013. Recuperado de: http://www.eldefensordelprofesor.es/wp-content/uploads/2015/11/ MEMORIA-DEFENSOR-DEL-PROFESOR-20122013_1385553981.pdf.

ANPE (2014). Memoria Estatal del Defensor del Profesor. Curso 2013-2014. Recuperado de: http://www.eldefensordelprofesor.es/wp-content/uploads/2015/02/dpmem1314. pdf.

Aparici, R. (2010). Comunicación y Web 2.0. En R. Aparici (coord.), Conectados en el ciberespacio (pp. 27-36). Madrid: UNED.

Área, M. (2012). La alfabetización en la sociedad digital. En M. Área, A. Gutiérrez y F. Vidal, Alfabetización digital y competencias informacionales (pp. 3-40). Madrid: Ariel.

Área, M. (2015). La alfabetización digital en la formación de la ciudadanía del siglo XXI. Revista Integra Educativa, 7(3), 21-33.

Área, M. y Pessoa, T. (2012). De lo sólido a lo líquido: las nuevas alfabetizaciones ante los cambios culturales de la web 2.0. Comunicar, 19, 13-20.

Área, M., Gutiérrez, A. y Vidal, F. (2012). Alfabetización digital y competencias informacionales. Madrid: Fundación telefónica.

Área, M., Gros, B. y Marzal, M. A. (2008). Alfabetizaciones y TIC. Madrid: Síntesis.

Armstrom, T. (2000). Multiple Intelligences in the classroom. Alexandria: ASCD.

Arnaiz, P. (2003). Educación inclusiva: una escuela para todos. Málaga: Aljibe.

Asensio, J., Álvarez, I., Vega, F. y Rodríguez, T. (2012). Las competencias de los profesionales de la Educación Hoy. La transformación de la práctica educativa. En XXXI Seminario Interunivesitario de Teoría de la Educación. Plasencia: Universidad Nacional de Educación a Distancia. Recuperado de: http://www.uned.es/site2012/ Ponencias.html.

Ballester-Vila, M. G. y Sánchez-Santamaría, J. (2012). La dimensión pedagógica del enfoque de competencias en educación obligatoria. Ensayos, Revista de la Facultad de Educación de Albacete, 26, 17-34.

Bauman, Z. (2006). Modernidad líquida. Buenos Aires: Fondo de Cultura Económica.

Beltrán, J. (2012). La educación inclusiva. Padres y Maestros. Publicación de la Facultad de Ciencias Humanas y Sociales, 338, 5-9. Recuperado de: http://revistas. upcomillas.es/index.php/padresymaestros/article/view/431/349.

Bisquerra, R. (2003). Educación emocional y competencias básicas para la vida. Revista de Investigación Educativa (RIE), 21(1), 7-43.

Bisquerra, R. (2005). La educación emocional en la formación del profesorado. $R e-$ vista interuniversitaria de formación del profesorado, 19(3), 95-114.

Bisquerra, R. y Pérez-Escoda, N. (2007). Las competencias emocionales. Revista de Educación Siglo XXI, 10, 61-82.

Booth, T., Ainscow, M. y Kingston, D. (2006). Index para la Inclusión: desarrollo del juego, el aprendizaje y la participación en educación infantil. Salamanca: INICO.

Bozu, Z. y Canto, P. J. (2009). El profesorado universitario en la sociedad del conocimiento: competencias profesionales docentes. Revista de Formación e Innovación Educativa Universitaria, 2(2), 87-97.

Brotheridge, C. M. y Grandey, A. A. (2002). Emotional labor and burnout: comparing two perspectives of "people work". Journal of vocational behavior, 60(1), 17-39. 
Cabello, R., Fernández-Berrocal, P. y Ruiz-Aranda, D. (2010) Docentes emocionalmente inteligentes. Revista Electrónica Interuniversitaria de Formación del Profesorado, 13(1), 41-49.

Castejón, J. L., Martínez, M. A. (2011). Competencias socioemocionales en el desarrollo profesional del maestro. Educación XX1, 14(2), 237-260.

Castells, M. (1997). La era de la información: economía, sociedad y cultura. Madrid: Alianza.

Castells, M. (2005). La era de la información: economía, sociedad y cultura: la sociedad Red. México: Alianza.

Castells, M. (2008). Creatividad, innovación y cultura digital. Un mapa de sus interacciones. Telos: Cuadernos de comunicación e innovación, 77, 50-52.

Castells, M. (coord.) (2006). La sociedad red: una visión global. Madrid: Alianza.

Chamarro, A., Cladellas, R., Longas, J. y Riera, J. (2012). La incidencia del contexto interno docente en la aparición del síndrome del quemado por el trabajo en profesionales de la enseñanza. Revista de Psicología del Trabajo y de las Organizaciones, 28(2) 107-118.

Cornejo, R. y Quiñonez, M. (2007). Factores asociados al malestar/bienestar docente. Una investigación actual. Revista Electrónica Iberoamericana sobre Calidad, Eficacia y Cambio en Educación, 5, 75-80.

Crespi, A. y Cañabate, A. (2010). ¿Qué es la sociedad de la información? Cátedra Telefónica-UPC. Análisis de la evolución y Tendencias Futuras de la Sociedad de la Información. Barcelona: Cátedra Telefónica-UPC.

De la Torre, C. (2007). El malestar docente: un fenómeno de relevancia internacional. Docencia e Investigación: Revista de la Escuela Universitaria de Magisterio de Toledo, 17, 301-325.

De Pablos, J. (2010). Universidad y sociedad del conocimiento. Las competencias informacionales y digitales. Revista de Universidad y Sociedad del Conocimiento (RUSC), 7, 2-12.

Demarzo, M., García, J., Gómez, B., Montero, J. y Pereira, J. (2013). Utilidad de los marcadores biológicos en la detección precoz y prevención del síndrome de burnout. Revista de Psicopatología y Psicología Clínica, 18(3), 245-253.

Diekstra, R. E. W. (2008). Evaluación de los programas escolares universales de educación emocional y social y de habilidades para la vida. En C. Clouder (coord.), Educación Emocional y social. Análisis Internacional. Informe Fundación Marcelino Botín 2008 (pp. 268-329). Santander: Fundación Marcelino Botín.

Durán, A., Extremera, N. y Rey, L. (2010). Recursos personales, síndrome de estar quemado en el trabajo y sintomatología asociada al estrés en docentes de enseñanza primaria y secundaria. Ansiedad y estrés, 16(1), 47-60.

Durán, M., Extremera, N., Montalbán, F. y Rey, L. (2005). "Engagement" y "burnout" en el ámbito docente: análisis de sus relaciones con la satisfacción laboral y vital en una muestra de profesores. Revista de Psicología del Trabajo y de las Organizaciones, 21(1-2), 145-158.

Durlak, J. A., Weissberg, R. P., Dymnicki, A. B., Taylor, R. D. y Schellinger, K. B. (2011). The impact of enhancing students social and emotion learning: a meta-analysis of school-based universal interventions. Child Development, 82, 405-432. 
Echeita, G. (2006). Educación para la inclusión o educación sin exclusiones. Madrid: Narcea.

Echeíta, G. (2016). Inclusión y exclusión educativa. De nuevo," Voz y quebranto". REICE. Revista iberoamericana sobre calidad, eficacia y cambio en educación, 11(2), 100-118.

Extremera, N. y Pena, M. (2012). Inteligencia emocional percibida en el profesorado de primaria y su relación con los niveles de burnout e ilusión por el trabajo (engagement). Revista de Educación, 359, 604-627.

Extremera, N., Fernández-Berrocal, P. y Durán, M. A. (2003). Inteligencia emocional y "burnout" en profesores. Encuentros en Psicología Social, 1, 260-265.

Extremera, N., Durán, A. y Rey, L. (2010). Perceived emotional intelligence and dispositional optimism-pessimism: Analyzing their role in predicting psychological adjustment among adolescents. Personality and Individual Differences, 42, 1069-1079.

Extremera, N., Pena, M. y Rey, L. (2012). Life satisfaction and engagement in elementary and primary educators: Differences in emotional intelligence and gender. Revista de Psicodidáctica, 17(2) 341-358.

Extremera, N., Rey, L. y Pena, M. (2010). La docencia perjudica seriamente la salud. Análisis de los síntomas asociados al estrés docente. Boletín de Psicología, 100, 43-54.

Fernández-Berrocal, P. y Ruíz-Aranda, D. (2008). La educación de la inteligencia emocional desde el modelo de Mayer y Salovey. En Acosta (dir.), Educación emocional y convivencia en el aula. Madrid: Ministerio de Educación y Ciencia.

Fernández-Berrocal, P. y Ruiz-Aranda, D. (2008a). La inteligencia emocional en la Educación. Revista Electrónica de Investigación Psicoeducativa, 15(6,2), 421-436.

Fernández-Montalvo, J., Peñalva, A. e Irazabal, I. (2015). Hábitos de uso y conductas de riesgo en Internet en la preadolescencia. Comunicar, 44, 113-120.

Ferragut, M. y Fierro, M. (2012). Inteligencia emocional, bienestar personal y rendimiento académico en preadolescentes. Revista Latinoamericana de Psicología, 44(3), 95-104.

García-Renedo, M., Llorens, S. y Salanova, M. (2005). Burnout como consecuencia de una crisis de eficacia: un estudio longitudinal en profesores de secundaria. Revista de Psicología del Trabajo y de las Organizaciones, 21(1-2) 54-70.

Gil-Monte, P. y Peiró, J. M. (1999). Validez factorial del Maslach burnout Inventory en una muestra multiocupacional. Psicothema, 11, 679-689.

Gil-Monte, P. y Peiro, J. M. (1997). Desgaste psíquico en el trabajo: Síndrome de quemarse. Madrid: Síntesis.

Jiménez-Morales, M. I. y López-Zafra, E. (2009). Inteligencia emocional y rendimiento escolar: estado actual de la cuestión. Revista Latinoamericana de Psicología, 41(1), 69-79.

Lévy, P. (2007). Cibercultura. La cultura de la sociedad digital. México: Anthropos.

Llorens, S. y Salanova, M. (2008). Estado actual y retos futuros en el estudio del burnout. Papeles del psicólogo, 29(1), 59-67.

Marina, J. A., Pellicer, C. y Manso, J. (2015). Libro Blanco de la profesión docente y su entorno escolar. Recuperado de: http://www.mecd.gob.es/mecd/dms/mecd/ destacados/libro-blanco/libro-blanco-profesion-docente.pdf. 
Martínez, A. E., Piqueras, J. A. e Inglés, C. (2014). Relaciones entre Inteligencia Emocional y estrategias de afrontamiento ante el estrés. Recuperado de: http://reme. uji.es/articulos/numero37/article6/article6.pdf.

Martínez, A. E., Piqueras, J. A. y Ramos, V. (2010). Inteligencia emocional en la salud física y mental. Electronic Journal of Research in Educational Psychology, 21(8,2), 861-890.

Maslach, C. (1997). Inventario burnout de Maslach (MBI): Síndrome del quemado por estrés laboral asistencial. Madrid: TEA.

Mayer, J. D., Salovey, P. y Caruso, D. R. (2002). Mayer-Salovey-Caruso Emotional Intelligence Test (MSCEIT). Canada: MHS Publishers.

Montolío, R. y Cervellera, L. (2008). Una escuela de Todas (las personas) para Todas (las personas). REICE: Revista Electrónica Iberoamericana sobre Calidad, Eficacia y Cambio en educación, 6(2), 100-119.

Montoya, A., Moreno, S. y Zuluaga, P. (2012). Relación entre síndrome de burnout, estrategias de afrontamiento y engagement. Psicología desde el Caribe, 29(1), 205-227.

Muñoz de Morales, M. (2005). Prevención del estrés psicosocial el profesorado mediante el desarrollo de competencias emocionales. Revista Interuniversitaria de Formación del Profesorado, 54, 115-136.

Pena, M. y Extremera, N. (2012). Inteligencia emocional percibida en profesorado de Primaria y su relación con los niveles de burnout e ilusión por el trabajo (engagement). Revista de Educación, 359, 604-627.

Pena, M. y Repetto, E. (2008). Estado de la investigación en España sobre la inteligencia emocional en el ámbito educativo. Revista Electrónica de Investigación Psicoeducativa, 15(6,2), 400-420.

Peñalva, A., López-Goñi, J. J. y Landa, N. (2013). Competencias emocionales del alumnado de Magisterio: posibles implicaciones profesionales. Revista de Educación, 362, 690-712.

Peñalva, A., López-Goñi, J. J. y García-Manso, I. (2016). El desarrollo de las competencias emocionales en alumnado de secundaria: perfiles diferenciales en función del sexo. En prensa.

Pérez-Gómez, A. I. (2010). Nuevas exigencias y escenarios para la profesión docente en la era de la información y la incertidumbre. Revista Interuniversitaria de Formación del Profesorado, 68, 17-36.

Pineda, M. (2011). Nuevas formas de ciudadanía asociadas a las redes de comunicación globales: el ciudadano digital. Historia Actual Online, 24, 163-183.

Premsky, M. (2010). Nativos e inmigrantes digitales. Institución educativa SEK. Recuperado de: http://www.marcprensky.com/writing/Prensky-NATIVOS\%20E\%20 INMIGRANTES\%20DIGITALES\%20\%28SEK\%29.pdf.

Pyhältö, K., Pietarinen, J. y Salmela-Aro, K. (2011). Teacher-Working-Environment Fit as a Framework for Burnout Experienced by Finnish Teachers. Teaching and Teacher Education, 27, 1101-1110.

Salanova, M., Schaufeli, W. B., Llorens, S., Peiró, J. M. y Grau, R. (2000). Desde el "burnout" al "engagement": una nueva perspectiva. Revista de Psicología del Trabajo y de las Organizaciones, 16, 117-134. 
Salmurri, F. (2004). Libertad emocional: Estrategias para educar las emociones. Madrid: Círculo de Lectores.

Salmurri, F. (2012). Avaluar les competències emocions a secundària. Escales per a la avaluació de les habilitats emocionals EHE-1. Barcelona: Fundació Propedagògic.

Salmurri, F. y Skoknic, V. (2005) Efectos conductuales de la educación emocional en alumnos de educación Básica. Revista psicológica de la Universidad de Chile, 14(1), 9-28.

Salovey, P. y Mayer, J. D. (1990). Emotional intelligence. Imagination, Cognition and Personality, 9, 185-211.

Salovey, P., Bedell, B., Detweiler, J. B. y Mayer, J. D. (2000). Current directions in emotional intelligence research. En M. Lewis y J. M. Haviland-Jones (eds.), Handbook of Emotions ( $2^{\circ}$ ed.) (pp. 504-520). New York: Guilford Press.

Sánchez, M. T., Fernández-Berrocal, P., Montañés, J. y Latorre, J. S. (2008). ¿Es la inteligencia emocional una cuestión de género? Socialización de las competencias emocionales en hombres y mujeres y sus implicaciones. Revista Electrónica de Investigación Psicoeducativa, 15(6,2), 455-474.

Tello, J. y Aguaded, J. I. (2009). Desarrollo profesional docente ante los nuevos retos de las tecnologías de la información y la comunicación en los centros educativos. Pixel-Bit. Revista de Medios y Educación, 34, 31-47.

UNESCO (2008). Inclusive education: the way of the future. Geneva: UNESCO.

Uztarroz, J. (2012). Las escuelas democráticas. Documentación Social, 163, 125 163.

Van Dijk, T. (2006). Discurso y manipulación: discusión teórica y algunas aplicaciones. Revista signos: estudios de lingüística, 60, 49-74.

Zins, J. E. (2004). Building academic success on social and emotional learning: what does the research says? New York: Teachers College Press. 